\title{
Limit-cycle-induced frequency locking in self-sustained current oscillations in superlattices
}

\author{
Z. Z. Sun, H. T. He, J. N. Wang, Shi-dong Wang, and X. R. Wang* \\ Physics Department, The Hong Kong University of Science and Technology, Clear Water Bay, Hong Kong, China \\ (Received 25 June 2003; revised manuscript received 15 September 2003; published 26 January 2004)
}

\begin{abstract}
The ac response of self-sustained current oscillations (SSCO's) in weakly coupled GaAs/AlAs superlattices (SL's) is derived based on the deformation of a limit cycle under an external ac driving force. Frequency locking into an integer fraction of the ac frequency is obtained in a periodic response in which a limit cycle deforms either with or without a topological change. This frequency locking is robust against the ac bias because a limit cycle can adjust itself. The results are verified both numerically and experimentally, indicating that SSCO's in SL's can be understood within the framework of the general concepts and principles of nonlinear physics.
\end{abstract}

DOI: 10.1103/PhysRevB.69.045315

PACS number(s): 73.50.Fq, 73.40.Gk, 73.61.-r

Following the early pioneering study ${ }^{1,2}$ on vertical electron transport in superlattices (SL's), one of the recent surprising discoveries is self-sustained current oscillations (SSCO's) under a dc bias. ${ }^{3,4}$ A large number of experimental and theoretical studies have focused on different aspects of these oscillations. Experimentally, it is known that SSCO's can be induced by varying the doping density, ${ }^{3}$ temperature, and magnetic field. ${ }^{4}$ Theoretically, it is understood that SSCO's are accompanied by the motion of boundaries of electricfield domains (EFD's). ${ }^{5}$ A model capable of describing both the formation of stationary EFD's and SSCO's emerged after many tedious analyses and numerical calculations. ${ }^{6}$ Our understanding of SSCO's was greatly advanced through numerical investigations of this model. As we know, a physical system with an intrinsic frequency may have various possible responses to an external driving force. For a linear system, such as a simple pendulum, it will oscillate with the frequency of the driving force. For a nonlinear system, however, it can oscillate with an integer multiple of the driving frequency. For example, a laser light passing through a nonlinear optical medium may lead to the second- and thirdharmonic generations. It is also known that many other nonlinear systems can have frequency locking, in which a system might oscillate with an integer fraction of the driving frequency. It is therefore interesting to ask how the SSCO's observed in SL's respond to a combined dc and ac bias. Numerical solutions on several SL models ${ }^{7-10,14}$ show possible aperiodic oscillations, either quasiperiodic or chaotic. The experimental evidences of chaotic behavior were also reported. ${ }^{11,12}$ While most early studies focused on the chaotic behavior of tunneling current, there were also studies of the response to an ac bias based on dipole EFD model. ${ }^{13,14}$ Many of the results were explained based on the concept of EFD and the motion of charge monopole or dipole. ${ }^{5,14-16}$ The question that we would like to ask is whether one can understand the SSCO's from an angle based on general fundamental concepts in nonlinear science.

In a recent study, ${ }^{17}$ we found that SSCO's in a SL can also be explained by the limit-cycle concept. In the terminology of nonlinear physics, ${ }^{18}$ SSCO's are the manifestation of onedimensional attractors-limit cycles. The power of the limitcycle concept lies in its simplicity and universality. An important question one might ask is if the SSCO's indeed come from limit cycles, what will be the possible responses of the SSCO's to an extra ac bias? As will be demonstrated in this paper, the EFD's model independent responses of SSCO's in SL's to an extra ac bias can be readily derived based on limit cycles. One will see that in periodic responses of SSCO's in SL's the frequency locking is not only a natural outcome of a limit cycle, but also very robust against the ac driving force. Furthermore, we can predict quantitatively the frequency of a periodic response for a given external ac bias. Thus, it is beneficial to use the limit cycle to understand SSCO's. First, by considering a limit cycle as a basic object, we argue that the limit cycle can have three possible responses to an extra ac bias: (1) a small deformation without a topological change, (2) a small deformation with a topological change, and (3) destruction of the limit cycle. The first two scenarios lead to the phenomenon of frequency locking, and the last one gives rise to an aperiodic response. Second, a widely used drift velocity model is solved numerically to demonstrate this type of frequency locking and its robustness. Finally, we present our experimental results. In experiment, we focus on the study of SSCO response to an external ac bias in weakly coupled GaAs/AlAs SL's. Both the periodic frequency response of a SSCO to an ac bias and the change in its wave forms under different ac bias are investigated. A two-dimension phase diagram is obtained in $I$ versus $d I / d t$ plane. It verifies the two distinct deformations adopted by ac driven limit cycles. An excellent agreement between the theory and the experiment is achieved.

For the sequential electron tunneling in a SL, the phase space is made up by the bias on each potential barrier, as explained in Ref. 17, since the state of a given SL is fully determined by these biases. On each point in the phase space, there is a unique vector which describes the system velocity in the space. ${ }^{18}$ This velocity is determined by the dynamics of the system. A point with zero velocity is called a fixed point. An unstable fixed point, as denoted by the cross in Fig. 1, is such that a small deviation from the fixed point will drive the system away from the point. However, the system will stay around the fixed point because of the external bias constraint. In the case of a SSCO, this local repulsion and global attraction lead the system to move along a closed curve, a limit cycle, around the fixed point. ${ }^{17,19}$ Using a two-dimensional case as an example, it is schematically 


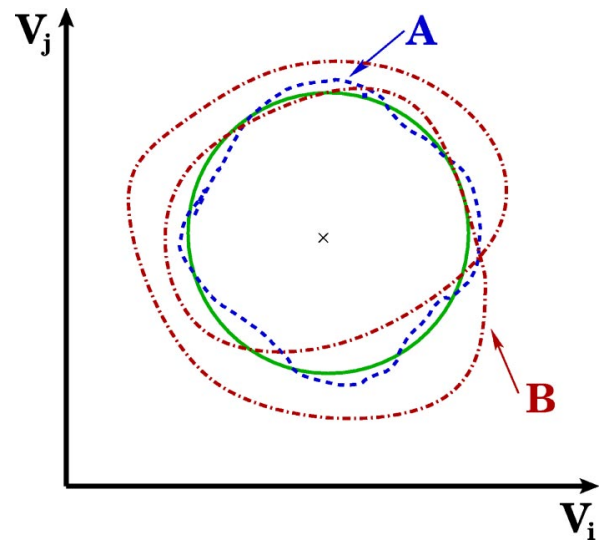

FIG. 1. Schematic drawing of limit cycles around an unstable fixed point (cross) in a phase plane. The loop in solid line is the limit cycle in the absence of an ac bias. Curves A and B are the two possible deformations of a limit cycle under an ac bias. The system shall oscillate with a frequency close to its intrinsic one in case A while it oscillates with half of the intrinsic frequency in case B.

illustrated in Fig. 1 as a loop in solid line. Applying an extra ac bias with frequency $\omega_{a c}$, the velocity field in the phase space changes through the dynamical equations of the system. If the bias is small, it can only perturb the velocity slightly, which, in turn, modifies the limit cycle.

For the periodic response, a limit cycle can change in two distinct ways. One way is a small deformation of the limit cycle without a topological change, as shown by the curve A in Fig. 1. The length of the limit cycle can at most change a little. The time period for a system to move along the closed curve once does not change substantially, since its velocity field in the phase space is controlled by the system dynamics that is perturbed only slightly by the extra ac bias. In this case, the system oscillates with a frequency $\omega$, whose value is not too far from its intrinsic frequency $\omega_{0}$. There is another requirement for the periodic response. Considering the system starting initially from a point on a limit cycle, it moves along the limit cycle and returns to the starting point after a time $T$, giving a frequency $\omega=2 \pi / T$. To have a periodic motion, the external ac bias should also return to its initial value. This means that $T$ must be an integer multiple of the ac-bias period $2 \pi / \omega_{a c}$. Thus, we have $\omega_{a c} / \omega=p$ $=$ integer. In fact, this is a general condition for the periodic motion of a dynamical system under an ac driving force. ${ }^{18} \mathrm{~A}$ natural conclusion of this argument is that the limit cycle makes a small deformation like that of curve A in Fig. 1, when the ac frequency $\omega_{a c}$ is in the vicinity of $p \omega_{0}$, an integer number of the system intrinsic frequency.

The other way is a topological change of the limit cycle in spite of slight perturbation of the phase velocity field. This can occur when the system trajectory does not close itself after moving around the fixed point once. Instead, the trajectory returns to its starting point after $q$ rounds. For example, the curve B in Fig. 1 shows a closed curve after two rounds. In this case, the system oscillates with a frequency around $\omega_{0} / 2$. It should be pointed out that this situation can occur only when the dimensionality of the phase space is larger than two. Combining the periodicity requirement discussed above, the second scenario occurs only when $\omega_{a c} / \omega_{0}$ is in the vicinity of $p / q$ ( $\neq$ integer), where $p$ and $q$ are integers. Under this type of ac bias, the limit cycle deforms itself in such a way that it becomes a closed curve after $q$ turns in the phase space, giving $\omega_{a c} / \omega=p$.

Obviously, the periodic response must oscillate with an integer fraction of the ac frequency if the SSCO's are indeed due to the generation of limit cycles around an unstable fixed point. This type of frequency locking does not depend on a particular model. Since the limit cycle can deform itself under an ac bias, this periodic response with an integer fraction of the ac frequency is expected to be quite robust against $\omega_{a c}$, meaning that $\omega_{a c} / \omega$ should remain unchanged with a small variation of $\omega_{a c}$. In order to verify these results, we numerically solve a widely used discrete drift model under the combined dc and ac biases in the SSCO's regime. For a system consisting of $N$ quantum wells under a bias $U$ between the two end wells, the current flow is perpendicular to the SL layers. In the sequential tunneling, charge carriers are in local equilibrium within each well, so that a chemical potential can be defined locally. The chemical potential difference between two adjacent wells is called bias $V$ on the barrier between the two wells. A current $I_{i}$ passes through the $i$ th barrier under a given bias $V_{i}$. This current may depend on other parameters, such as doping $N_{D}$.

Following Refs. 5 and 17, the dynamics of the system is governed by the discrete Poisson equations

$$
k\left(V_{i}-V_{i-1}\right)=n_{i}-N_{D}, \quad i=1,2, \ldots, N
$$

and the current continuity equations

$$
J=k \frac{\partial V_{i}}{\partial t}+I_{i}, \quad i=0,1,2, \ldots, N,
$$

where $k$ depends on the SL structure and its dielectric constant. $n_{i}$ is the electric charge in the $i$ th well. In Eq. (1), the same doping in all wells is assumed. $I_{i}$ is, in general, a function of $V_{i}$ and $n_{i}$. It can be shown ${ }^{20}$ that all SSS's are stable if $I_{i}$ is a function of $V_{i}$ only. This result can be understood as follows. The dynamic system can be decoupled into $N$ onedimensional dynamic systems with a parameter $J$. General nonlinear theory ${ }^{18}$ guarantees at least one stable SSS. On the other hand, a SSS may be unstable ${ }^{5}$ if one chooses $I_{i}$ $=n_{i} v\left(V_{i}\right)$, where $v$ is a phenomenological drift velocity which is, for simplicity, assumed to be a function of $V_{i}$ only. The constraint equation for $V_{i}$ is

$$
\sum_{i=0}^{N} V_{i}=U
$$

To close the equations, a suitable boundary condition is needed. It is reasonable to assume a constant $n_{0}, n_{0}$ $=\delta N_{D}$, if the carrier density in the emitter is much larger than those in wells, and its change due to a tiny tunneling current is negligible.

Previous studies ${ }^{5,17}$ have shown that this model is capable of describing SSCO's with a negative differential drift velocity. One can obtain a SSCO when $v(V)=0.0081 /[(V / E$ $\left.-1)^{2}+0.01\right]+0.36 /\left[(V / E-2.35)^{2}+0.18\right], \quad N=30, \quad U$ 


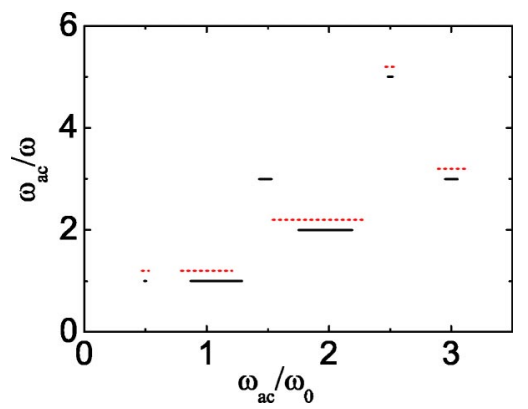

FIG. 2. $\omega_{a c} / \omega$ vs $\omega_{a c} / \omega_{0}$. The devil's staircase type behavior with $\omega_{a c} / \omega=p$, with integer $p$, shows that the system oscillates with an integer fraction of ac frequency, and this response is robust against $\omega_{a c}$. Solid line is for $V_{a c}=0.327 E$ and dashed line is for $V_{a c}=0.654 E$. Staircases with very narrow widths are not displayed.

$=32.7 E, N_{D}=0.095 k E$, and $\delta=1.001$ are used. ${ }^{17}$ The drift velocity is the sum of two Lorentz functions. This function form is justified if the negative differential resistance is due to the resonance tunneling between two subbands in the wells of SL's. This $v$ has two peaks at $V=E$ and $V$ $=2.35 E$. The region from $V=E$ to $V=1.3 E$ exhibits negative differential velocity. Thus, $E$ can be used as a natural unit of bias, and $1 / v(E)$ as that of the time (the lattice constant is set to be 1). The intrinsic frequency $\omega_{0}$ is $0.14[v(E) / 1]$, indicating that the corresponding EFD boundary oscillates inside about seven wells. Now we apply an extra ac bias $V_{a c} \sin \left(\omega_{a c} t\right)$ with $V_{a c}=0.01 U$ in addition to the above dc bias. Also, we have solved numerically the above set of equations for different $\omega_{a c}$. The current oscillation frequency can be obtained from the Fourier transformation of time evolution of the current. The results are plotted in the $\omega_{a c} / \omega$ versus $\omega_{a c} / \omega_{0}$ plane shown by the solid line in Fig. 2. It has a structure similar to a devil's staircase. The width of the staircase contains the information of robustness that the limit cycle can adjust itself. This robustness depends on both the amplitude and the frequency of ac bias. The width of the devil's staircase around $\omega_{a c} / \omega_{0}=1 / q$ decreases with the increase of $q$ and the results are not displayed for $q$ $\geqslant 3$. The dashed line is the similar result for $V_{a c}=0.02 U$. The data are offset vertically for a better view. Clearly, the periodic response around $\omega_{a c}=1.5 \omega_{0}$ disappears, leading to a possible chaotic response. It shows that the limit cycle is destroyed, and the phase trajectory, somehow, cannot make a closed curve under this ac bias.

To verify the above results of frequency locking and its robustness experimentally, we have measured the response of SSCO's under an extra ac bias from a GaAs/AlAs SL sample. The GaAs/AlAs SL sample is grown by molecular beam epitaxy. It consists of 30 periods of $14 \mathrm{~nm}$ GaAs well and $4 \mathrm{~nm}$ AlAs barrier and is sandwiched between two $n^{+}$-GaAs layers. The central $10 \mathrm{~nm}$ of each GaAs well is doped with $\mathrm{Si}\left(n=2 \times 10^{17} \mathrm{~cm}^{-3}\right)$. The sample is fabricated into $0.2 \times 0.2 \mathrm{~mm}^{2}$ mesas. The SSCO's response is recorded using an Agilent infiniium 54832B oscilloscope. It has been found that SSCO's in a SL can be induced by changing the sample temperature. ${ }^{4}$ In this measurement, the sample tem-

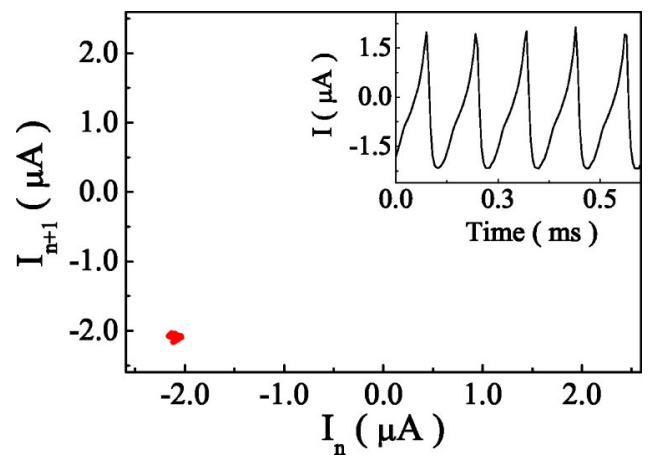

FIG. 3. The original time dependence of tunneling current (a) and the corresponding Poincare map: the points are $\left[I\left(n T_{0}\right)\right.$, $\left.I\left(n T_{0}+T_{0}\right)\right]$ for $n=1,2, \ldots$ (b). The accumulation of all points into a single dot in this map indicates that the current oscillates periodically with a period of $T_{0}\left(=2 \pi / \omega_{0}\right)$.

perature is fixed at $100 \mathrm{~K}$ and the dc bias at $0.42 \mathrm{~V}$, which is located within the first plateau of the time-averaged $I-V$ curve.

Figure 3(a) shows the current oscillation trace without any ac bias. The SSCO's are clearly demonstrated with a frequency $\omega_{0}=27.2 \mathrm{KHz}$. The oscillation periodicity is indicated by the well-formed single dot in the corresponding Poincaré map (or the first return map), ${ }^{18}$ as shown in Fig. 3(b). The Poincare map is derived from the current oscillation trace by sampling the current trace in a step of $T_{0}$ $\left(=2 \pi / \omega_{0}\right)$. It is noted that a Poincaré map is very sensitive to the time interval used. If the time interval is not commensurable to the periodicity of a periodically oscillated curve, the map obtained consists of curves or lines instead of a few isolated dots. One needs to choose the time interval in such a way that there are only isolated spots in Poincaré maps.

Figure 4 shows Poincaré maps obtained with an applied extra ac bias applied to the SSCO shown in Fig. 3. The ac bias amplitude $V_{a c}$ is set at $29 \mathrm{mV}$ and the driving frequencies $\omega_{a c}$ are indicated. The Poincare maps are obtained with sampling steps $T_{a c}\left(=2 \pi / \omega_{a c}\right)$. As discussed early in this paper, the system exhibits the frequency locking when $\omega_{a c}$ is set in the vicinity of $p \omega_{0}$ or $\omega_{0} / p$ with $p=$ integer. The corresponding response period $T$ is given by the number of

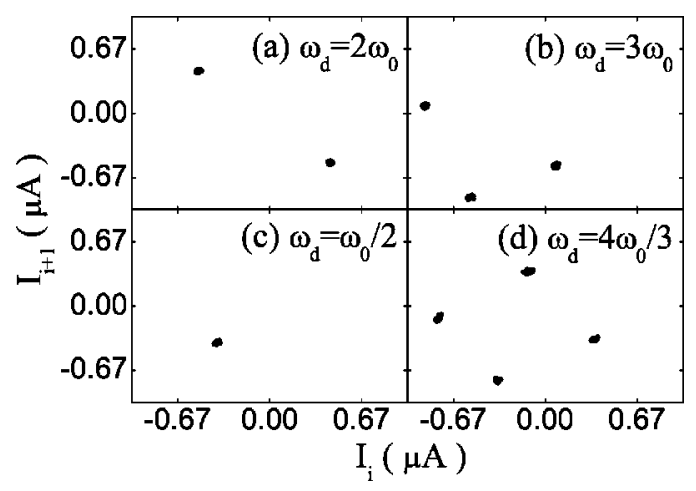

FIG. 4. Poincaré maps with sampling step $T_{a c}$ for $\omega_{a c}=2 \omega_{0}$ (a), $\omega_{a c}=3 \omega_{0}$ (b), $\omega_{a c}=\omega_{0} / 2$ (c), $\omega_{a c}=4 \omega_{0} / 3$ (d). The number of dots in the maps multiplying $T_{a c}$ are the corresponding response periods. 


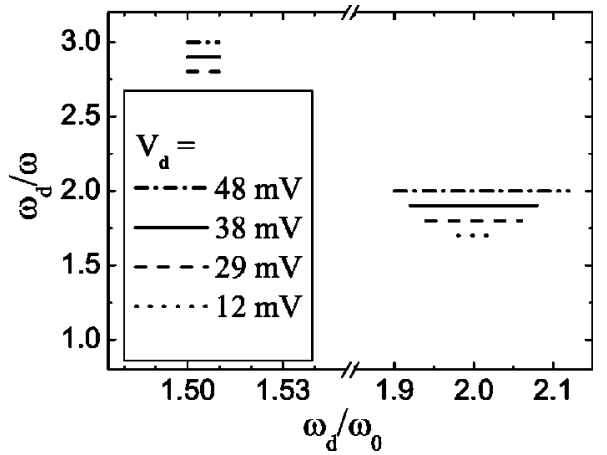

FIG. 5. Experimentally measured periodic response ranges around $\omega_{a c} / \omega_{0}=1.5$ and 2 , respectively, illustrated in $\omega_{a c} / \omega$ vs $\omega_{a c} / \omega_{0}$ plots for different amplitudes of ac bias indicated. The data are offset for clarity.

dots in each case multiplying $T_{a c}$. The measured response frequency $\omega$ is equal to $\omega_{a c} / p$ for $\omega_{a c}=p \omega_{0}$ or $\omega_{a c}$ for $\omega_{a c}=\omega_{0} / p$, respectively. The accumulation of all sampled points into several discrete dots, as shown in Figs. 4(a)-4(d), clearly demonstrates the occurrence of the frequency locking when $\omega_{a c}=2 \omega_{0}, 3 \omega_{0}, \omega_{0} / 2$, and $4 \omega_{0} / 3$, respectively. As a result, for $\omega_{a c}=2 \omega_{0}$ and $3 \omega_{0}$ [see Figs. 4(a) and 4(b)], $T$ is given as $2 T_{a c}$ and $3 T_{a c}$, respectively, which are both equal to $T_{0}$. On the other hand, when $\omega_{a c}=\omega_{0} / 2$ and $4 \omega_{0} / 3$ [see Figs. 4(c) and 4(d)], the response periods are $T_{a c}\left(=2 T_{0}\right)$ and $4 T_{a c}\left(=3 T_{0}\right)$, respectively. Frequency locking is also observed for other integer fraction of ac bias frequency.

In order to demonstrate the robustness of the frequency locking, we focus on the frequency locking in the vicinity of $\omega_{a c}=3 \omega_{0} / 2$ and $2 \omega_{0}$. The existence of a locking range in the vicinity of each $p \omega_{0} / q$ is observed, indicating the robustness of the frequency locking. As long as $\omega_{a c}$ is set in this locking range, frequency locking with the same response frequency $\omega=\omega_{a c} / p$ is obtained. In Fig. 5 experimental results in the vicinity of $3 \omega_{0} / 2$ and $2 \omega_{0}$ are plotted to demonstrate this robustness. By varying the applied ac bias frequency and amplitude, the existence of the devil's staircase is explicitly shown, in which the data for different ac bias amplitude $V_{a c}$ are offset vertically for clarity. The lengths of the lines in Fig. 5 indicate the frequency locking range around $\omega_{a c} / \omega_{0}=1.5$ and 2 for a given $V_{a c}$. Clearly, the devil's staircase widths, i.e., the robustness of frequency locking, are strongly dependent on $V_{a c}$ and $\omega_{a c}$. The locking range for $\omega_{a c} / \omega_{0}=2$ is much larger than that for $\omega_{a c} / \omega_{0}$ $=1.5$. When $V_{a c}$ is small $(=12 \mathrm{mV})$ the periodic response cannot be found around $\omega_{a c} / \omega_{0}=1.5$. These results are in good agreement with the theoretical expectations.

Besides the above periodic frequency response of a SSCO to an ac bias, its wave form characteristics in the presence of frequency locking are also investigated. Curves (a)-(d) shown in Fig. 6 are the oscillation current traces for $\omega_{a c}$ $=n \omega_{0}, n=1,2,3$, and 4, respectively, with $V_{a c}=29 \mathrm{mV}$. For comparison, the free current trace without any ac bias applied is also plotted in Fig. 6. Only one prominent peak appears within each period (indicated by arrow) of free SSCO trace. When an external ac bias is applied some extra wiggles are superimposed on the main peak, as shown in

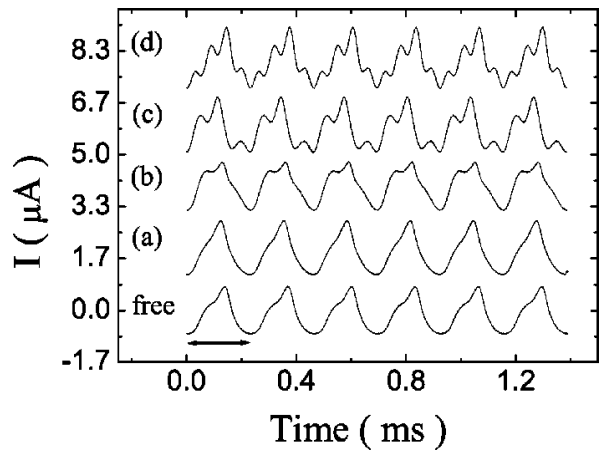

FIG. 6. The real time current traces for $\omega_{a c}=\omega_{0}$ (a), $\omega_{a c}$ $=2 \omega_{0}$ (b), $\omega_{a c}=3 \omega_{0}$ (c), $\omega_{a c}=4 \omega_{0}$ (d). The "free" one is the original current oscillation trace without any ac bias applied.

curves (a)-(d) of Fig. 6. When $n$ increases, the number of these wiggles also increases but the response frequency in each case remains the same. In contrast, the corresponding current traces exhibit another type of change in wave forms for the cases with $\omega_{a c}=\omega_{0} / n$. In Fig. 7 curves (a) and (b) are the current traces for $\omega_{a c}=\omega_{0} / 2$ and $\omega_{0} / 3$, respectively, plotted together with the free one for comparison. The $V_{a c}$ is also set to $29 \mathrm{mV}$. An arrow indicates the oscillation period for each case. In comparison with the free one, the ac-driven current traces exhibit more than one prominent peak within each period. Two peaks are observed for $\omega_{a c}=\omega_{0} / 2$ [see curve (a) in Fig. 7] and three peaks for $\omega_{a c}=\omega_{0} / 3$ [see curve (b) in Fig. 7].

To further demonstrate that the frequency locking and SSCO wave form variations can indeed be understood in terms of the limit-cycle theory, $I$ versus $d I / d t$ phase diagrams are obtained from the free and ac-driven current traces shown in Figs. 6 and 7. These phase diagrams are twodimensional projections of the limit cycles. As an illustration, the limit cycles for $\omega_{a c}=2 \omega_{0}$ (open triangles) and $\omega_{a c}=\omega_{0} / 2$ (open circles) are shown in Figs. 8(a) and 8(b), respectively. The free limit cycle without any ac bias applied is plotted for comparison [see solid squares in Figs. 8(a) and $8(\mathrm{~b})]$. When an ac bias with $\omega_{a c}=2 \omega_{0}$ is applied, the free limit cycle evolves into a new limit cycle [see curve A in Fig.

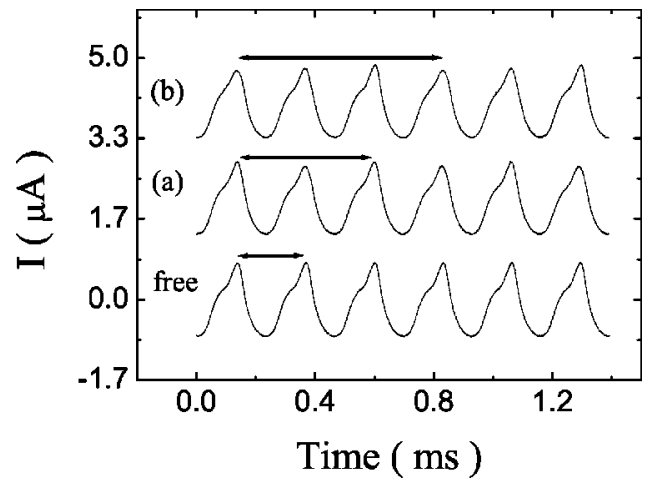

FIG. 7. The real time current traces for $\omega_{a c}=\omega_{0} / 2(\mathrm{a}), \omega_{a c}$ $=\omega_{0} / 3(\mathrm{~b})$. The "free" one is the original current oscillation trace without any ac bias applied. The arrows indicate the period of each current trace. 


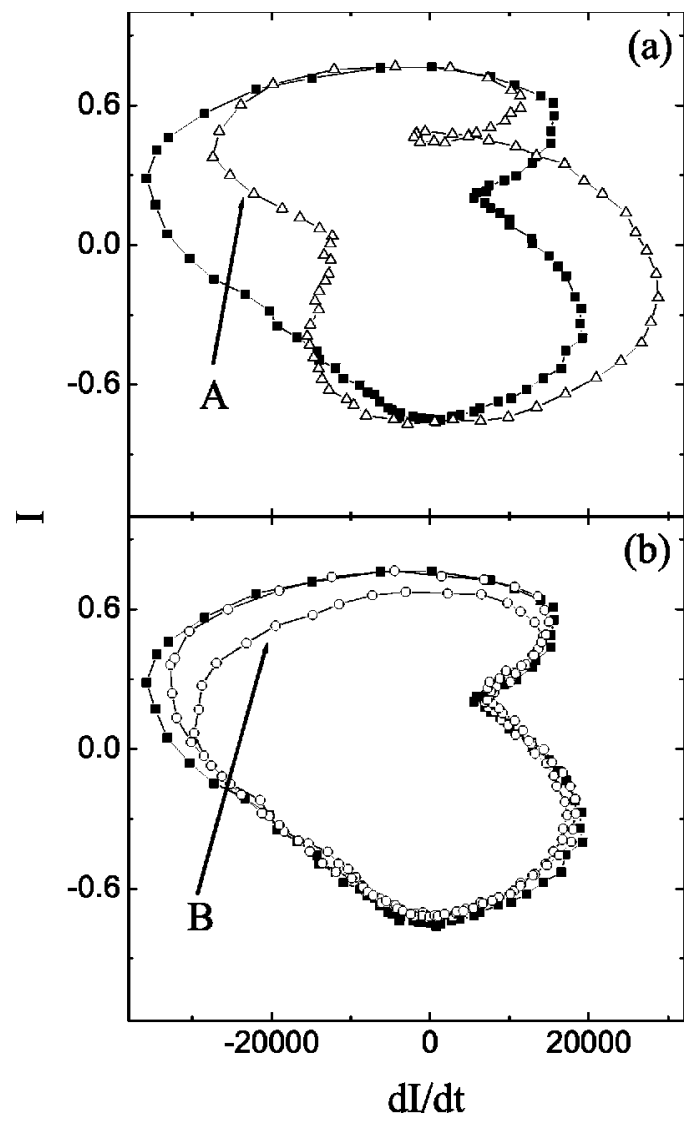

FIG. 8. Schematic drawing of limit cycles in a phase plane. The close square loop is the limit cycle in the absence of any ac bias. Curve A and B represent the two distinct deformation ways of the limit cycles, A for $\omega_{a c}=2 \omega_{0}$ and B for $\omega_{a c}=\omega_{0} / 2$.

$8(\mathrm{a})]$ by changing its shape. This new ac-driven limit cycle closes itself after one round giving rise to the frequency locking with response frequency $\omega=\omega_{0}=\omega_{a c} / 2$. In contrast, when $\omega_{a c}$ is set to $\omega_{0} / 2$ the free limit cycle experiences a topological change [see curve B in Fig. 6(b)]. The resulting new limit cycle returns to its initial point after two rounds. This results in the frequency locking with response frequency $\omega=\omega_{0} / 2=\omega_{a c}$. The observed two types of deformations of limit cycle agrees well with the theory discussed earlier.

These two types of deformations can also be used to explain the observed wave form characteristics [see Figs. 6 and 7]. For the deformation without topological change the ac forced limit cycle A in Fig. 8(a) moves one round for each period. This one-round motion generates one prominent peak in each period of the current trace [see curve (b) in Fig. 6]. The extra wiggles superimposed on this main peak originate from the deformation of the shape of limit cycle in comparison with the free one. As for the deformation with a topological change the limit cycle B in Fig. 8(b) moves two rounds for each period. Each time when B moves a round a large current peak is generated [see curve (a) in Fig. 7].
Since the shape of limit cycle varies slightly for each round motion and is similar to the free one the two current peaks in each period also change slightly. The above analysis is generally applicable to all the cases for $\omega_{a c}=n \omega_{0}$ and $\omega_{a c}$ $=p \omega_{0} / q$.

The above demonstrated quantitative agreements among the theory, the model calculation, and the experiment clearly indicate that a SSCO is indeed the manifestation of a limit cycle around an unstable fixed point in the phase space. The system moves along this limit cycle round by round resulting in the SSCO. The unusual frequency locking into a particular set of an integer fraction of ac frequency is the direct manifestation of deformation of limit cycles under ac bias. It is worth to emphasize that based on limit cycles frequency locking does not depend on the particular structure of the EFD. Like many other nonlinear dynamical systems, the frequency locking of SSCO's can be understood within the framework of the general concepts and principles of nonlinear physics. We believe this view of frequency locking applicable to some other nonlinear systems. However, one should view current limit-cycle theory and previous EFD theory to be complementary to each other. The previous theory ${ }^{15,16}$ gives a microscopic origin of SSCO's in combination of front dynamics and the injection condition of the contact. The limit cycle provides a way to visualize this behavior and is of great help for understanding the more complicated situation under additional ac bias.

In summary, the periodic response of a SSCO to an external ac bias is investigated both theoretically and experimentally. The limit-cycle theory offers a good explanation for the experimental results. The limit cycle can deform itself in such a way that it makes $q$ turns in the phase space around an unstable fixed point when the ac frequency $\omega_{a c}$ is in the vicinity of $(p / q) \omega_{0}$ with integers $p$ and $q$. Thus, a system may oscillate with $\omega_{a c} / p$, an integer fraction of $\omega_{a c}$, or about $\omega_{0} / q$. Both of this type of frequency locking into an integer fraction of the driving ac frequency and its robustness are verified by the numerical model calculations and real experiments. According to the current traces phase diagrams are plotted in $I$ versus $d I / d t$ plane corresponding to a twodimensional projection of a limit cycle. It reveals that under an extra ac bias a new limit cycle is formed by the deformation with or without a topological change in comparison with that without any ac bias applied. In conclusion, the limitcycle picture of SSCO's gives a deep insight into the nonlinear properties of SL's.

This work was supported by UGC, Hong Kong, through Grants Nos. HKUST6149/00P, HKUST6162/01P, and 603403. X.R.W. thanks Professor P. Tong for useful discussions. We are very grateful to Professor Yiping Zeng for providing SL samples. We would like to thank high school student helper, Mr. Henry Hongjia Wang, for checking references.
*Author to whom correspondence should be addressed. Electronic
address: phxwan@ust.hk
${ }^{1}$ L. Esaki and R. Tsu, IBM J. Res. Dev. 14, 61 (1970); L. Esaki and L.L. Chang, Phys. Rev. Lett. 33, 495 (1974). 
${ }^{2}$ M. Büttiker and H. Thomas, Phys. Rev. Lett. 38, 78 (1977).

${ }^{3}$ S.H. Kwok, T.B. Norris, L.L. Bonilla, J. Galán, J.A. Cuesta, F.C. Martínez, J.M. Molera, H.T. Grahn, K. Ploog, and R. Merlin, Phys. Rev. B 51, 10171 (1995); H.T. Grahn, J. Kastrup, K. Ploog, L.L. Bonilla, J. Galán, M. Kindelan, and M. Moscoso, Jpn. J. Appl. Phys. 34, 4526 (1995); J. Kastrup, R. Klann, H.T. Grahn, K. Ploog, L.L. Bonilla, J. Galán, M. Kindelan, M. Moscoso, and R. Merlin, Phys. Rev. B 52, 13761 (1995).

${ }^{4}$ J.N. Wang, B.Q. Sun, X.R. Wang, and H.L. Wang, Solid State Commun. 112, 371 (1999); Jiannong Wang, Baoquan Sun, Xiangrong Wang, Yuqi Wang, Weikun Ge, and Hailong Wang, Appl. Phys. Lett. 75, 2620 (1999); X.R. Wang, J.N. Wang, B.Q. Sun, and D.S. Jiang, Phys. Rev. B 61, 7261 (2000).

${ }^{5}$ J. Kastrup, R. Hey, K.H. Ploog, H.T. Grahn, L.L. Bonilla, M. Kindelan, M. Moscoso, A. Wacker, and J. Galán, Phys. Rev. B 55, 2476 (1997); A. Wacker, M. Moscoso, M. Kindelan, and L.L. Bonilla, ibid. 55, 2466 (1997); G. Schwarz and E. Schöll, Phys. Status Solidi B 194, 351 (1996); M. Patra, G. Schwarz, and E. Schöll, Phys. Rev. B 57, 1824 (1998).

${ }^{6}$ L.L. Bonilla, J. Galán, J.A. Cuesta, F.C. Martínez, and J.M. Molera, Phys. Rev. B 50, 8644 (1994); F. Prengel, A. Wacker, and E. Schöll, ibid. 50, 1705 (1994); A. Wacker, F. Prengel, and E. Schöll, in Proceedings of the 22nd International Conference on the Physics of Semiconductors, edited by D. J. Lockwood (World Scientific, Singapore, 1995), Vol. 2, p. 1075; R. Aguado, G. Platero, M. Moscoso, and L.L. Bonilla, Phys. Rev. B 55, R16 053 (1997).

${ }^{7}$ O.M. Bulashenko and L.L. Bonilla, Phys. Rev. B 52, 7849 (1995); O.M. Bulashenko, M.J. García, and L.L. Bonilla, ibid. 53, 10008 (1996).

${ }^{8}$ J.C. Cao and X.L. Lei, Phys. Rev. B 60, 1871 (1999); J.C. Cao, H.C. Liu, and X.L. Lei, ibid. 61, 5546 (1999).
${ }^{9}$ A. Amann, J. Schlesner, A. Wacker, and E. Schöll, Phys. Rev. B 65, 193313 (2002); E. Schöll, Nonlinear Spatio-Temporal Dynamics and Chaos in Semiconductors (Cambridge University Press, Cambridge, England, 2001).

${ }^{10}$ M. Zwolak, D. Ferguson, and M. Di Ventra, Phys. Rev. B 67, 081303(R) (2003).

${ }^{11}$ Yaohui Zhang, J. Kastrup, R. Klann, K.H. Ploog, and H.T. Grahn, Phys. Rev. Lett. 77, 3001 (1996).

${ }^{12}$ K.J. Luo, H.T. Grahn, K.H. Ploog, and L.L. Bonilla, Phys. Rev. Lett. 81, 1290 (1998); K.J. Luo, H.T. Grahn, S.W. Teitsworth, and K.H. Ploog, Phys. Rev. B 58, 12613 (1998); O.M. Bulashenko, K.J. Luo, H.T. Grahn, K.H. Ploog, and L.L. Bonilla, ibid. 60, 5694 (1999).

${ }^{13}$ E. Schomburg, K. Hofbeck, R. Scheuerer, M. Haeussler, K.F. Renk, A.-K. Jappsen, A. Amann, A. Wacker, E. Schöll, D.G. Pavel'ev, and Yu. Koschurinov, Phys. Rev. B 65, 155320 (2002); A.-K. Jappsen, A. Amann, A. Wacker, E. Schöll, and E. Schomburg, J. Appl. Phys. 92, 3137 (2002).

${ }^{14}$ D. Sánchez, G. Platero, and L.L. Bonilla, Phys. Rev. B 63, R201306 (2001).

${ }^{15}$ D. Sánchez, M. Moscoso, L.L. Bonilla, G. Platero, and R. Aguado, Phys. Rev. B 60, 4489 (1999); L.L. Bonilla, G. Platero, and D. Sánchez, ibid. 62, 2786 (2000); L.L. Bonilla, J. Phys.: Condens. Matter 14, R341 (2002).

${ }^{16}$ A. Wacker, Phys. Rep. 357, 1 (2002); A. Amann, A. Wacker, and E. Schöll, Physica B 314, 404 (2002).

${ }^{17}$ X.R. Wang and Q. Niu, Phys. Rev. B 59, R12 755 (1999); X.R. Wang, Z.Z. Sun, S.Q. Duan, and Shi-dong Wang, cond-mat/0211306 (unpublished).

${ }^{18}$ R. Seydel, Practical Bifurcation and Stability Analysis (SpringerVerlag, New York, 1994).

${ }^{19}$ Bai-Lin Hao, Chaos II (World Scientific, Singapore, 1990).

${ }^{20}$ Z. Z. Sun et al. (unpublished). 